\title{
Estudio Cualitativo sobre el Comportamiento del Consumidor en las Compras en Línea
}

\author{
Kathya E. Mercado ${ }^{1}$, Cynthia B. Perez ${ }^{2^{\star}}$, Luis A. Castro ${ }^{1}$ y Adrian Macias ${ }^{1}$ \\ (1) Instituto Tecnológico de Sonora, Campus Náinari, Antonio Caso S/N y E. Kino, Colonia Villa ITSON. C.P. \\ 85130. Ciudad Obregón, Sonora. México. (e-mail: kathya.mercado@hotmail.com; luis.castro@acm.org; \\ adrian.macias@itson.edu.mx) \\ (2) Instituto Tecnológico de Sonora, Unidad Guaymas, Carretera al Aeropuerto Km 3, C.P. 85400. Apartado \\ Postal 870. Guaymas, Sonora. México. (e-mail: cynthia.perez@itson.edu.mx)
}

Recibido May. 17, 2018; Aceptado Jul. 23, 2018; Versión final Sep. 14, 2018, Publicado Feb. 2019

\begin{abstract}
Resumen
Se estudia las características principales del comportamiento del consumidor en plataformas de compra en línea en el estado sur de Sonora. Esto se hace mediante un estudio cualitativo basado en la metodología de la Teoría Fundamentada. La idea principal es identificar y categorizar variables sobre la compra en línea basándose en factores relevantes como la motivación de compra, preferencias, hábitos de consumo y patrones de compra. Para llevar a cabo el estudio, se realizaron entrevistas semi-estructuradas a consumidores del estado sur de Sonora, México, con experiencia en compras en línea. De esta manera, al concluir el análisis, se obtuvieron tres categorías principales: motivación de compra, experiencias del consumidor y comportamiento. También se identificaron una serie de variables que tienen mayor influencia en las preferencias, decisión de compra y el patrón de compra en los consumidores. Los resultados obtenidos proporcionan información de utilidad para la creación de estrategias que permitan tomar decisiones efectivas mediante su aplicación en el diseño de plataformas en línea ofreciendo un servicio personalizado a los consumidores.
\end{abstract}

Palabras clave: análisis cualitativo; teoría fundamentada; compras en línea; comportamiento del consumidor; entrevista semi-estructurada

\section{Qualitative Study of Consumer Behavior in Online Shopping}

\begin{abstract}
The main characteristics of consumer behavior in online shopping platforms at Sonora, Mexico have been studied. A qualitative study based on the methodology of Fundamental Theory is used to identify and categorize variables about online purchases based on relevant factors such as purchase motivation, preferences, consumption habits and purchasing patterns. Semi-structured interviews to consumers with experience in online purchases were conducted. In this way, three main categories were obtained: purchase motivation, consumer experiences and behavior. Also, some variables with a greater influence on preferences, purchase decision and consumer purchase pattern were identified. The obtained results provide useful information for strategies planning that allow making effective decisions using them in the design of online platforms with a personalized service to consumers.
\end{abstract}

Keywords: qualitative analysis; grounded theory; online purchases; consumer behavior; semi-structured interview 


\section{INTRODUCCIÓN}

El imparable desarrollo y expansión de las nuevas tecnologías de la información, en especial en el comercio electrónico ha causado el creciente interés en el comportamiento del consumidor en compras en línea (Zhou et al., 2007). Asimismo, la sociedad actual demanda más comodidad y menos tiempo en la compra de productos o servicios por Internet, por lo cual, están conscientes que es el medio más rápido para la búsqueda de información y muestra la importancia de que las empresas tengan cada vez más capacitación para mantener un efectiva presencia en Internet (Gallaugher, 1997; Grandon y Pearson, 2004; Barrera, 2017). El comercio electrónico consiste en la compra-venta de productos o servicios, en donde los consumidores obtienen información y adquieren productos a través de medios electrónicos (Olson y Olson, 2000). Por esta razón, Zarco y Ruiz (2006), plantean que la estructura comercial, el sistema de distribución y los canales de distribución han tenido considerables transformaciones en los últimos años, de modo que han sido ampliamente promocionados los beneficios potenciales del uso del comercio electrónico (Gefen y Straub, 2000). Sin embargo, para que se obtengan estos beneficios se necesita examinar la interacción del consumidor en el proceso de compra en una plataforma de compra en línea (Pavlou y Fygenson, 2006).

Hoy en día, el estudio del comportamiento del consumidor ha sido ampliamente estudiado desde diferentes perspectivas y áreas (Solomon et al., 2014). La mayoría de los estudios se han centrado principalmente en la acción de compra de productos o servicios y medir la satisfacción del consumidor, enfocándose en las experiencias posteriores a la compra de los consumidores con el fin de encontrar una relación con variables que enriquezcan más el conocimiento acerca del consumidor (Westbrook et al., 1991; Homburg y Annette, 2001). Por otra parte, existen varios estudios, que se centran en el análisis de datos tanto cuantitativos como cualitativos, para demostrar la relación entre la motivación de los consumidores y su comportamiento de compra (Joines et al., 2003; Goldsmith y Horowitz, 2006). Por ejemplo, Hausman (2000), probó que la decisión de compra de los consumidores es una consecuencia de los impulsos generados por la motivación a las recompensas que se obtendrán por realizar una compra. Asimismo, Karbasivar (2011), demuestra que existe una relación fundamental entre la publicidad de productos, las actividades promocionales (descuentos, productos o servicios gratuitos) y el comportamiento de compra del consumidor. Por lo cual, se afirma que el comportamiento del consumidor no es monolítico, puesto que, antes de realizar una compra los consumidores primero participan en la obtención de información de un producto o servicio y en otros factores que proporciona el sitio web. De modo que se plantean retos de investigación relacionados con el análisis y modelización del comportamiento del consumidor en las compras en línea, por ejemplo, 1) ¿Cuáles son os factores más relevantes para la comprensión del comportamiento del consumidor? Y 2) ¿Cómo se relacionan entres si los factores encontrados?, usando los resultados como la base principal sobre la que se formulan estrategias organizacionales.

En este artículo, se presenta un estudio cualitativo bajo el enfoque de la Teoría Fundamentada para estudiar el comportamiento, actitudes y percepciones de los consumidores que compran productos y servicios en línea. Para esto, el objetivo del estudio es identificar las variables del comportamiento del consumidor de mayor relevancia en el proceso de decisión de compra en línea por medio del análisis de entrevistas de consumidores que realizan compras de productos y servicios en línea. Dentro de esas variables consideramos la interpretación y medición de la relación de los diferentes factores relevantes para las compras en línea, tales como la percepción de los productos, motivación de compra, preferencias, hábitos de consumo, influencias y comportamientos de compra. Asimismo, con la finalidad de identificar si el proceso de decisión de compras se ha visto alterado y cuáles han sido las variables del comportamiento del consumidor que han sido influenciadas. Los resultados obtenidos de esta investigación demuestran la importancia de 3 categorías para definir el comportamiento del consumidor en las compras en línea las cuales son: 1) la motivación de compra, 2) las experiencias, y 3) el comportamiento del consumidor. Además, se presenta cómo la motivación y la experiencia influyen en el comportamiento del consumidor.

\section{METODOLOGIA}

La Teoría Fundamenta nos permite construir teorías, hipótesis y conceptos acerca de un fenómeno en particular, partiendo directamente de los datos mediante la utilización de un método comparativo constante (Glaser y Strauss, 1967). En este trabajo se realizó un estudio cualitativo para analizar e identificar las variables del comportamiento del consumidor de mayor relevancia en el proceso de decisión de compra.

\section{Preguntas de investigación}

Este proyecto de investigación se basó en tres preguntas enfocadas a consumidores de productos y servicios en línea: P1. ¿Cuáles son las prácticas de compras por Internet que ejercen los consumidores?; P2. ¿Cuáles son los motivos por lo que los consumidores compran a través de Internet?; y P3. ¿Qué caracteriza la experiencia y el comportamiento de consumidores en sus compras por Internet? 


\section{Informantes}

El estudio se llevó a cabo en el sur del estado de Sonora, en México, donde participaron personas adultas con experiencia en compras en línea. Para el proceso de selección, los participantes debían cumplir los siguientes criterios de elegibilidad: residir en el sur del estado de Sonora, tener entre 18 y 45 años de edad, contar con experiencia en compras en línea, tener suficiente capacidad de destreza cognitiva, visual y manual para el uso de la computadora o dispositivo móvil, tener acceso diario a una computadora personal con conexión a Internet y contar con cualquier nivel ingreso, educación y ocupación. Para el proceso de selección de participantes, se realizó una breve entrevista telefónica a catorce personas para determinar la elegibilidad de los participantes. Al finalizar las entrevistas telefónicas, se seleccionaron once personas que cumplieron con los criterios de elegibilidad quienes se comprometieron a participar en el estudio, ver Tabla 1. En esta tabla se presentan los participantes seleccionados y sus características en cuanto a la edad, ocupación y sexo donde se seleccionaron 4 de las entrevistas realizadas, dos de estudiantes (P5 y P10) y dos profesionistas (P2 y P3).

Tabla 1: Descripción de participantes.

\begin{tabular}{|c|l|c|c|}
\hline No. Participante & \multicolumn{1}{|c|}{ Ocupación } & Edad & Sexo \\
\hline P1 & Estudiante de Posgrado & 24 & $\mathrm{~F}$ \\
\hline P2 & Técnico en Electrónica & 35 & $\mathrm{M}$ \\
\hline P3 & Ejecutivo de Ventas & 42 & $\mathrm{~F}$ \\
\hline P4 & Técnico de Sistemas & 25 & $\mathrm{M}$ \\
\hline P5 & Estudiante de Posgrado & 23 & $\mathrm{~F}$ \\
\hline P6 & Estudiante de Posgrado & 36 & $\mathrm{M}$ \\
\hline P7 & Estudiante de Carrera & 20 & $\mathrm{~F}$ \\
\hline P8 & Desarrollador Web & 24 & $\mathrm{M}$ \\
\hline P9 & Profesor de Español & 33 & $\mathrm{M}$ \\
\hline P10 & Estudiante de posgrado & 27 & $\mathrm{M}$ \\
\hline P11 & Contador Público & 32 & $\mathrm{M}$ \\
\hline
\end{tabular}

Con el objetivo de determinar un adecuado tamaño muestral para este trabajo de investigación, se empleó el método del muestreo selectivo basado en la Teoría Fundamentada donde la estructuración de la muestra de los participantes se realiza gradualmente a lo largo del proceso, por lo que el número y los rasgos de la población no se conocen a priori. Por ello, Glaser y Strauss (1967) definen que en este tipo de muestro la elección de individuos a estudiar se produce durante el proceso de interpretación de datos. Asimismo, Flick (2012) considera que el muestreo teórico puede partir de personas específicas donde la extensión de la población no se conoce, el tamaño de la muestra no está definido y el muestro acaba cuando se alcanza la saturación teórica, a diferencia del muestreo estadístico donde todo esto se conoce a priori. Glaser y Strauss (1967), definen como saturación teórica el criterio para determinar cuándo dejar de muestrear los distintos individuos, grupos o casos. Por lo tanto, se considera que se ha llegado a la saturación cuando no se hallan datos, o bien, cuando ya no emerge nada nuevo (Valles, 2009). De esta manera, la muestra no se obtiene a través de un muestreo aleatorio, sino que los individuos son seleccionados mediante las expectativas que generan en el aporte a ideas nuevas en relación con la teoría que se desea desarrollar. Adicionalmente, es importante considerar el tiempo disponible para llevar a cabo la investigación cualitativa debido a que el análisis de los datos cualitativos recolectados es muy rigoroso y toma demasiado tiempo para llevarlo a cabo de forma manual. Para este estudio, se recolectaron once entrevistas de las cuales se analizaron cuatro, debido a que se encontró saturación teórica, tomando un tiempo de tres meses para la codificación y análisis de las cuatro entrevistas. Así mismo, con la codificación de los datos y el análisis de los mismos se consideró que la información recogida resultó suficiente en relación con el objetivo del estudio.

\section{Protocolo de entrevista}

Los datos se obtuvieron de las entrevistas realizadas a los participantes. Cada una de las entrevistas semiestructuradas con preguntas abiertas fueron conducidas por el investigador con cada participante para documentar sus experiencias sobre las compras realizadas por Internet. Para esto, la entrevista se dividió en bloques de preguntas para facilitar el análisis, se obtuvieron diez bloques con diferentes temas a tratar: compras por Internet en general, acerca de su última compra realizada, compras en tiendas extranjeras, servicio de paquetería seleccionado, formas de pago utilizadas, tipo de dispositivos en los que realizan compras en línea, la confianza, la utilidad y control percibido a la hora de realizar compras en línea, la utilidad de la información proporcionada en los sitios de compras en línea, ver Tabla 2. 
Tabla 2: Estructura de la guía de entrevista.

\begin{tabular}{|l|c|}
\hline \multicolumn{1}{|c|}{ Bloques de preguntas } & $\begin{array}{c}\text { Número de } \\
\text { preguntas }\end{array}$ \\
\hline Acerca de compras en línea & 9 \\
\hline Acerca de su última compra & 9 \\
\hline Compras en tiendas extranjeras & 8 \\
\hline Utilidad de los sitios de compra por internet & 5 \\
\hline Control percibido & 10 \\
\hline Envío de productos & 6 \\
\hline Formas de pago & 9 \\
\hline Tipo de dispositivos en las compras por internet & 9 \\
\hline Confianza percibida & 5 \\
\hline Utilidad de la información de un sitio de compra en línea & 10 \\
\hline
\end{tabular}

La entrevista consistió en llevar a cabo preguntas generales de inicio y posteriormente profundizar con preguntas cada vez más específicas como por ejemplo: “¿Con qué regularidad compras en línea?”, “¿Qué es lo que más valoras a la hora de comprar en línea?” y “¿Cuáles han sido tus experiencias en la compra en línea?”. Cada entrevista duró aproximadamente de 30 a 45 minutos, las cuales fueron transcritas para su posterior análisis. Una vez transcritas fueron codificadas de forma abierta acorde a la Teoría Fundamentada.

\section{Análisis de datos}

Se utilizó la técnica de Teoría Fundamentada, la cual consiste en un procedimiento sistemático para analizar y generar teorías a partir de datos cualitativos (Strauss y Corbin, 1998). Para Strauss et al. (2002), el propósito principal de la Teoría Fundamentada en los datos consiste en generar o encontrar modelos explicativos sobre determinados fenómenos, cuyos principios teóricos se encuentran apoyados en el análisis sistemático y posteriormente a la interpretación de los datos obtenidos de un conjunto de informantes mediante entrevistas. Por lo cual, se debe basar en el análisis comparativo constante entre las opiniones de los informantes en relación con la situación de cada pregunta con respecto a sus compras realizadas, con el fin de identificar patrones de recurrencia y sus relaciones. Como resultado del uso de esta metodología es la generación de teoría a partir de la inducción, de esta manera, ayuda a la explicación exacta del fenómeno estudiado aportando al investigador la habilidad de explicar un suceso. De esta manera, la aplicación de esta metodología permite crear teoría basada en la realidad, usando lo expresado por los informantes buscando mantener la esencia de las palabras que nos otorgaron.

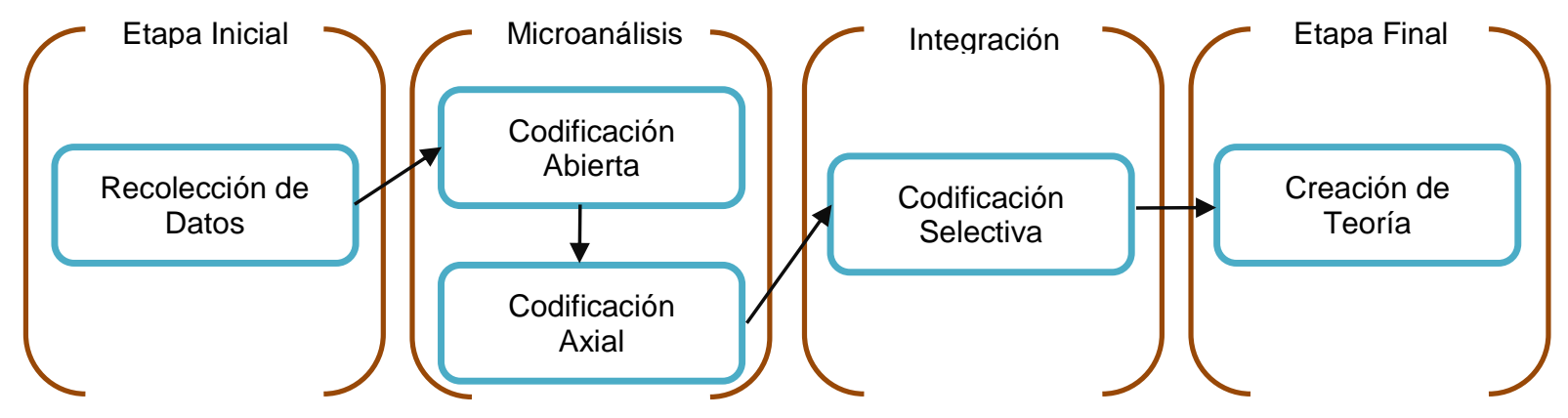

Fig. 1: Pasos de la Teoría Fundamentada

La metodología utilizada se basa en un modelo de cuatro etapas presentadas en la Figura 1. En este modelo se identifican las 4 etapas y 5 procesos a seguir para la creación de teoría. Primeramente en la etapa inicial se comienza con la identificación de área de interés a explorar y la selección de las fuentes de información, subsiguiente con la recolección de los datos. En la etapa de Microanálisis se comienza con el análisis de los datos y se van identificando que información relevante para el estudio, en esta etapa se realiza la codificación abierta y axial. La codificación abierta de los datos, se entiende como el análisis minucioso para identificar y conceptualizar los significados que el texto contiene. El resultado es una lista de códigos y al compararlos respecto de sus propiedades y dimensiones, se obtiene una clasificación, denominada categoría. Posteriormente, se lleva a cabo un análisis de codificación axial, en la cual, de acuerdo con Spiggle (1994), se identifican las relaciones entre las categorías obtenidas en la codificación abierta y sus subcategorías, esta relación está determinada por 
las propiedades y dimensiones de las subcategorías y categorías que se quieren relacionar; por ejemplo, el código A causa el código B, el código A confirma el código B.

Una vez llevada a cabo la etapa de microanálisis, se procede a la etapa de integración donde se realiza la codificación selectiva, la cual, es una extensión de la codificación axial, pero con un mayor nivel de abstracción. Su propósito es obtener una categoría central que exprese el fenómeno de investigación para desarrollar una única línea narrativa con la cual todas las demás categorías relacionadas con la central estén cubiertas (Locke, 2001). Finalmente, en la última etapa se lleva a cabo el proceso de la creación de teoría, en la cual se empieza a delimitar las teorías emergentes y se formula una teoría con un grupo pequeño de conceptos de alta abstracción que permitirán construir conocimiento basado en las experiencias aportadas por los informantes. Esta metodología exige un laborioso trabajo de selección de contenidos relevantes, en función de lo expresado por los participantes, lo cual implica un alto nivel de abstracción donde el investigador no debe perder el sentido subjetivo que el informante transmitió.

\section{Codificación abierta}

Para llevar a cabo la codificación abierta de los datos obtenidos mediante las entrevistas, se analizaron detalladamente las transcripciones de éstas, línea por línea para identificar códigos y definir las categorías mediante la agrupación de conceptos. En la Figura 2, se muestra la codificación abierta de la transcripción de la entrevista del participante dos (P2). De esta manera, se detectan códigos abiertos sobre los datos recogidos, por ejemplo, en la respuesta del participante dos se identificó un patrón de navegación, al indicar la utilización de un buscador en los sitios de compra en línea, a su vez, manifiesta la necesidad de un catálogo en los sitios de compras en línea y se detecta un problema durante la navegación. Estos códigos seleccionados sirvieron posteriormente para la identificación de patrones e información importante para la siguiente fase.

\begin{tabular}{|c|c|c|}
\hline & E2 & Yo utilizo el buscador y ya con eso me es suficiente pero no todos tienen \\
\hline 155 & & buscador eh! [Patrón de navegación] yo digo que deben tener un catálogo \\
\hline 156 & & que estén divido por categorías que sea simple y sencillo y que también \\
\hline 157 & & tengan un buscador [necesidades en compras]. Porque es muy difícil \\
\hline 158 & & navegar cuando no tienen y solo los dividen por marca [Problemas: difícil \\
\hline 159 & & navegación]. Por ejemplo si yo entro a Privalia no le voy poner una "blusa \\
\hline 160 & & roja" no, o sea es lo que este actual las ofertas actuales y ver todo el \\
\hline 161 & & catalogo que tienen. \\
\hline
\end{tabular}

Fig. 2: Codificación abierta: rotulación a entrevista

\section{Codificación Axial}

En esta fase del proceso de análisis se identificaron las relaciones entre las categorías obtenidas en la codificación abierta, en la cual se determinaron la variedad de condiciones, acciones/interacciones y consecuencias asociadas al comportamiento del consumidor en compras en línea. Por lo cual se llevó a cabo la jerarquización en subcategorías que son relacionadas con las categorías obtenidas previamente, ver Tabla 3.

Esta Tabla presenta el análisis y el desarrollo de las categorías relevantes para el estudio del comportamiento del consumidor, la cual está conformada por cuatro columnas: categorías, subcategorías, propiedades y dimensiones.

Tabla 3: Descripción de categorías, subcategorías, propiedades y dimensiones

\begin{tabular}{|c|l|l|l|}
\hline \multirow{4}{*}{ Categorías } & \multicolumn{1}{|c|}{ Subcategorías } & \multicolumn{1}{c|}{ Propiedades } & \multicolumn{1}{c|}{ Dimensiones } \\
\hline \multirow{4}{*}{ Comportamiento } & \multirow{2}{*}{ Patrón de compra } & $\begin{array}{l}\text { Frecuencia de } \\
\text { compras }\end{array}$ & Regularmente \\
\cline { 3 - 4 } & & \multirow{2}{*}{ Horario } & Ocasionalmente \\
\cline { 4 - 4 } & & Mañana \\
\cline { 3 - 4 } & & Tarde \\
\cline { 3 - 4 } & & Noche \\
\hline
\end{tabular}


Tabla 3 (continuación)

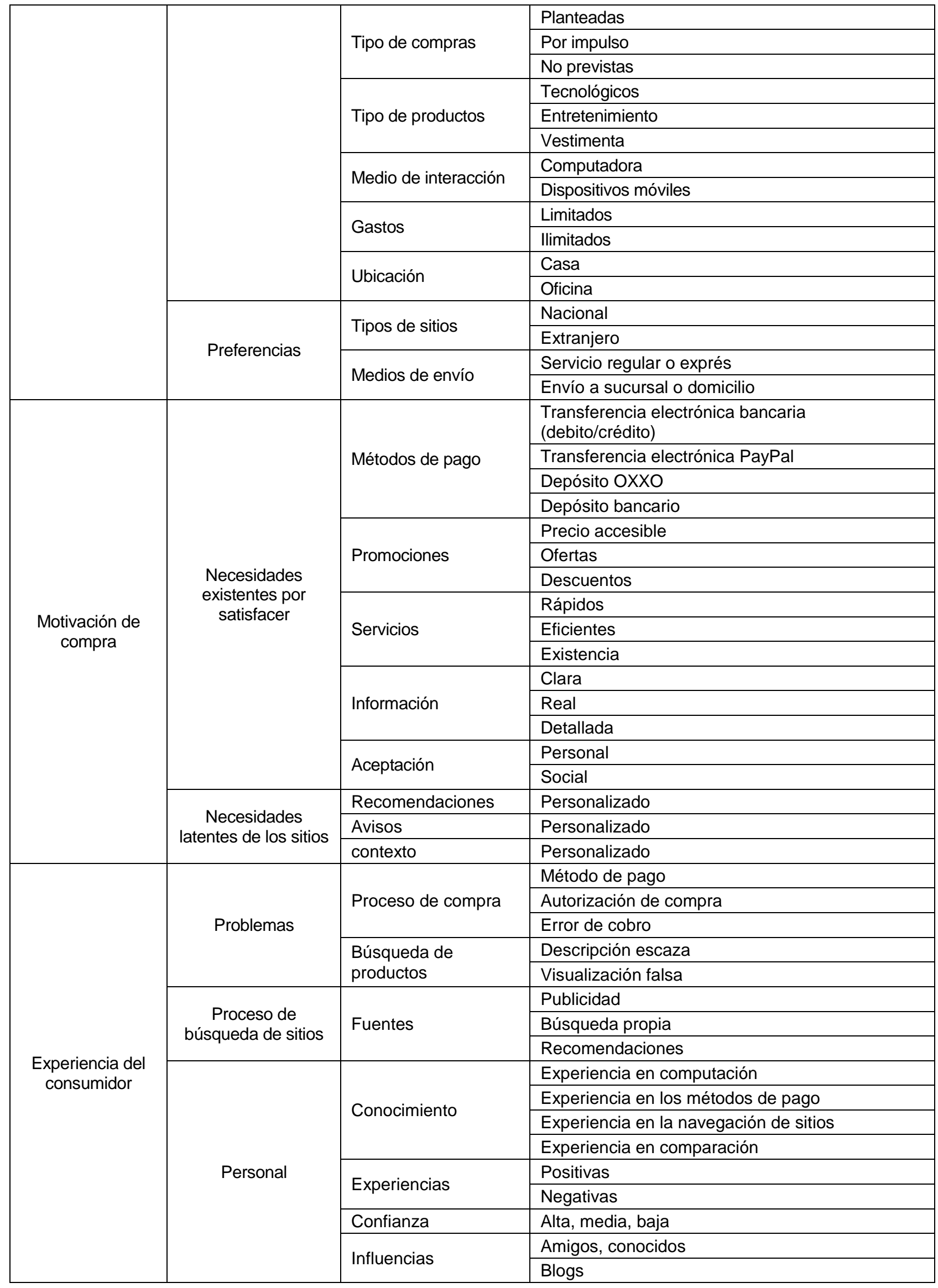

En la Tabla 3 se muestra una de las categorías que se definieron para la interpretación del estudio del comportamiento del consumidor, la cual nos define el comportamiento del consumidor y da a conocer las relaciones causales donde el comportamiento del consumidor genera un patrón de compra y otras subcategorías 
como lo son las preferencias del consumidor. Una vez que la categoría se identificó, se describieron sus propiedades y las características de dimensión.

\section{Codificación selectiva}

En esta fase del proceso se selecciona una categoría para ser el núcleo, y se relacionan todas las demás categorías con la central. La categoría central en este estudio es la del comportamiento presentada como una categoría en la Tabla 3. Así mismo, se describen otras dos categorías, la motivación y la experiencia del consumidor, las cuales están directamente relacionadas con la categoría central y explican las condiciones y consecuencias de la misma. En conjunto, las tres categorías ayudaron de manera significativa para la creación de teoría que explica el comportamiento del consumidor en compras en línea. La recolección y análisis de datos en el proceso de investigación ocurrió simultáneamente y la teoría que surgió al concluir el proceso de análisis de los datos. En el cual, los códigos obtenidos de la recolección y análisis de los datos fueron comparados e interpretados dentro del contexto de transcripciones generales presentados en la siguiente sección.

\section{RESULTADOS}

Los datos se presentaron utilizando el marco propuesto por Strauss y Corbin (1998) para el desarrollo de categorías y subcategorías. La codificación selectiva se concentró en la formulación de una categoría central para obtener la idea conceptual bajo la cual se agruparán todos los elementos de las categorías alrededor de la central. Las categorías seleccionadas y desarrolladas fueron: comportamiento, motivación de compra y experiencias del consumidor. Finalmente, se generaron ideas a partir de las relaciones de las categorías, las cuales dieron origen a la teoría que describe los factores que influyen en mayor medida el comportamiento del consumidor en compras en línea, presentada en la Figura 3. En la Figura 3 se muestra como la motivación y la experiencia influyen en el comportamiento del consumidor. Asimismo, se identifican las variables que conforman a cada uno de estas categorías. A continuación se describe cada uno de las categorías del modelo teórico - explicativo.

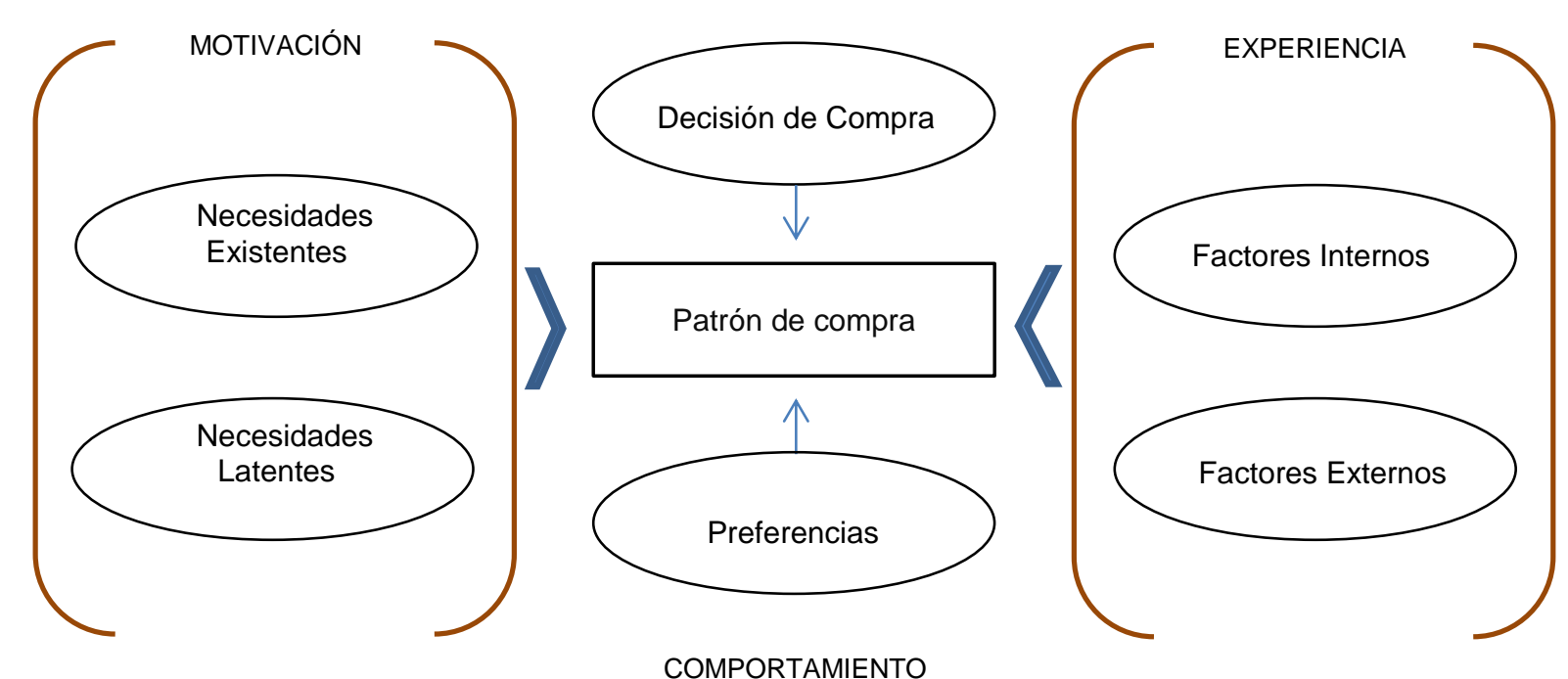

Fig. 3: Modelo teórico-explicativo del comportamiento del consumidor

\section{Categoría 1: Comportamiento}

Esta categoría es la principal del estudio, en ella se define el comportamiento del consumidor. Por lo cual, el comportamiento es definido por las actividades del consumidor orientadas a la compra de productos y servicios, incluyendo los procesos de decisión y sus preferencias, influenciadas por las dos subcategorías (motivación y experiencia) que preceden y determinan el patrón de compras del consumidor.

\section{Variable 1: Patrón de compras}

Con base en los comentarios de los participantes se identificó que durante los últimos años los consumidores adquieren productos y servicios mediante una mayor búsqueda de información y procesos de selección que tiene mucho que ver con sus preferencias de consumo sobre qué, dónde y cuánto comprar. Por lo tanto, los participantes manifestaron un alto empoderamiento de sus decisiones de compra como se observa en el comentario del participante 3 , en donde expone que son capaces de exigir mayores y mejores condiciones en sus compras en línea, realizando búsquedas previas en diferentes sitios de comercio electrónico para seleccionar 
el más adecuado a sus preferencias. Comentario del participante 3 (P3): "Pues depende que es lo que estoy comprando y en donde, por ejemplo, yo siempre por regla comparo los precios, nunca, nunca, nunca jaja, la verdad no compro la primera vez que entro al sitio, por ejemplo, la última compra que quería hacer la última semana... compare ese producto en las tiendas físicas, entonces ya sabía los precios de las tiendas que si tenía en mi ciudad y luego comparé en línea porque a veces ponen ofertas exclusivas en línea".

Asimismo, el comentario del P10 nos demuestra que no solo se limitan a obtener información de productos y servicios en general, sino que también analizan los comentarios de revisiones de otros consumidores que han adquirido un producto o servicio, esto indica que es una actividad que influye en gran medida en su decisión de compra. El comentario de este participante es el siguiente: "Al tener el sitio donde es más barato reviso los comentarios de los clientes que han comprado el mismo producto para ver si me decido comprar". Por otra parte, en el comentario del participante 10 se identifica una fase de exploración obligatoria, en donde sienten una responsabilidad de indagar, explorar y encontrar información para sentirse seguros al realizar su compra, tal como sigue: "Pues, yo creo depende de la personalidad de como sea la persona, por ejemplo yo soy muy paciente al buscar porque puedo durar 2 horas metida buscando artículos y porque quiero encontrar cosas que me gusten y estén baratas pero a mí se me hace muy fácil sobre todo si tiene los filtros para cambio rangos de precios, o por marcas y si esos filtros no los tuviera la verdad sería una flojera y así es más fácil encontrar algo que busco, mientras más detallado un filtro mejor y es lo que me ha gustado en los sitios que uso para comprar".

\section{Variable 2: Preferencias}

Con base en las experiencias de los participantes, se encontró que los consumidores tienen una lista priorizada de sus preferencias, como se muestra en los siguientes comentarios de los participantes 2, 3 y 5: "Las compras por las páginas chinas usan correos de México, si he comprado en páginas así y he pagado extra el envío y ha sido DHL para que me llegue rápido, pero sólo en páginas en donde ofrecen eso porque no todos los vendedores te lo ofrecen." (P3) "Uso más mercado libre por la rapidez de envío... lo que más valoró es la rapidez de entrega..." (P2) "También se me hace más seguro comprar en Amazon que en otro sitio, además que este sitio tiene el servicio de envío gratuito."(P5)

Por ejemplo, en cuanto a preferencias de los tipos de sitios, los medios de envío y los métodos de pago, están influenciadas directamente con las necesidades existentes por satisfacer y por la motivación de compra. Sin embargo, a la hora de la toma de decisión de compra se ven más inclinados a los medios de envío por encima de los precios y sitios de compra, como lo demuestra el comentario del participante 10: "Por ejemplo, un día compré en una página donde si tu pedido era mayor a los 70 dólares tu envío era gratis pero como yo quería que me llegará pronto me valió pagar extra por el pedido." (P10)

\section{Categoría 2: Motivación de compra}

Esta categoría influye directamente sobre el comportamiento de compra, siendo identificada como el estímulo de comprar en línea.

\section{Variable 3: Necesidades existentes por satisfacer}

En este estudio, se describió esos estímulos como necesidades existentes por satisfacer incluyendo las promociones, servicios, información y aceptación que proporcionan estos sitios en línea.

Refiriéndose a esto, los participantes declararon: "La variedad de los productos, que actualicen su catálogo de productos." (P5); "Quiero encontrar cosas que me gusten y estén baratas." (P10); y "Por los costos muy bajos y porque encuentras cosas que en otros sitios no encuentra."(P2)

\section{Variable 4: Necesidades latentes de los sitios}

También se detectaron necesidades relacionadas al medir, sentir o palpar el producto donde los participantes mostraron interés que deben ser atendidas de alguna manera para tener mejores experiencias de compra. A continuación comentarios del participante 10: “...la experiencia de medirse, sentirlo y ver cómo te queda no lo tienes y poner fotos reales puede acércanos un poco a esta experiencia de las tiendas físicas. También estaría padre tener a alguien ahí por la plataforma para poder preguntarle cosas sobre los productos." (P10)

\section{Categoría 3: Experiencias del consumidor}

En cuanto a la categoría de experiencias del consumidor, los participantes comentaron que es un factor importante para la definición del comportamiento de compra, debido a que los consumidores poseen conocimientos previos a la hora de navegar por sitios de compras en línea, esto indica que al momento de sentir el estímulo que anteriormente se define como motivación, la experiencia proporciona la información suficiente 
que influirá en su comportamiento de compra. Esta experiencia se dividió en problemas (variable 5), proceso de búsqueda (variable 6) y personal (variable 7) donde cada una de estas variables se explican a continuación.

\section{Variable 5. Problemas}

De acuerdo a los comentarios de los participantes, las compras en línea implican un acto de fe en el que el consumidor pone su confianza en los sitios de compras en línea, para que sus pedidos sean enviados en tiempo y forma. Sin embargo, todos han pasado por desafortunadas situaciones que hicieron no obtener su producto $o$ servicio y perder su dinero. Por lo cual, proporcionan sus experiencias y lecciones aprendidas de los eventos problemáticos a la hora de comprar, como lo expresa el siguiente comentario: "Lo debes hacer con moderación, checar muy bien el sitio que vas a utilizar, que sea un sitio web conocido, que tenga muchos usuarios y leer de perdida unos puntos de contrato de privacidad que te proporcionan algunos sitios." (P2)

\section{Variable 6. Proceso de búsqueda}

Con base a las experiencias de los participantes, se encontró que la distribución y existencia de los elementos básicos de navegación en un sitio de compras en línea es de vital importancia para la decisión de compra, como se muestra en el comentario del participante 3: "Que este CLARO todo, que este todo completo, lo que te presentan y ¡claro!. Que sea fácil y que el buscador ¡híjole!, el buscador es algo que se busca, bueno por lo menos yo es lo primero que uso cuando quiero algo en específico, pero a veces entro a páginas nomas para ver qué hay de nuevo..."(P3)

\section{Variable 7. Personal}

Del mismo modo, las experiencias buenas y malas son un factor clave para el comportamiento de consumidor, porque son las responsables de influir en mayor medida en las preferencias y decisión de compra de los consumidores. El comentario del participante 3 expresa tener una vasta experiencia en compras en línea y que la mayoría han sido buenas: "Mis experiencias son bastante buenas, son que encuentras siempre lo que buscas, es económico, no batallé en el proceso de compra, al momento de buscar y hacer el pedido." (P3). Por lo cual, a partir del establecimiento de las conexiones de las categorías relacionadas (motivación y experiencia) con la categoría central (comportamiento) se pueden enriquecer el conocimiento actual sobre el comportamiento del consumidor ante plataformas de compra en línea, mediante la implementación de estrategias de mejora con respecto a los resultados obtenidos, como se muestra en la Tabla 4.

Tabla 4: Estrategia de mejora en plataformas de compra en línea de acuerdo a las categorías definidas en el estudio

\begin{tabular}{|c|c|}
\hline Categorías & Estrategia de mejora en plataformas de compra en línea \\
\hline Motivación & $\begin{array}{l}\text { 1) Actualización constante del catálogo de productos y servicios. } \\
\text { 2) Mantener siempre descuentos y promociones. } \\
\text { 3) Contar una descripción de productos detallada, al igual que fotos de productos reales que se } \\
\text { pueden observar todas las características del producto. } \\
\text { 4) Proporcionar un chat online para resolver las inquietudes o miedos que le vayan surgiendo a los } \\
\text { consumidores de la plataforma de compra en línea en tiempo real. }\end{array}$ \\
\hline Comportamiento & $\begin{array}{l}\text { 5) Contar con una sección de comentarios para que los consumidores expresen sus opiniones } \\
\text { acerca de los productos y servicios consumidos y ayuden en la decisión de compra de futuros } \\
\text { consumidores. } \\
\text { 6) Proporcionar filtros de búsqueda de productos y servicios de diferentes tipos: marca, precio, } \\
\text { departamento, entre otros. } \\
\text { 7) Ofrecer diferentes medios de pago y paquetería. }\end{array}$ \\
\hline Experiencia & $\begin{array}{l}\text { 8) Proporcionar información acerca de garantías, políticas y contrato de privacidad. } \\
\text { 9) Contar con un buscador interno de productos. } \\
\text { 10) Ofrecer información acerca de devoluciones y reembolso. } \\
\text { 11) Contar con una sección de contacto para solución de problemas. }\end{array}$ \\
\hline
\end{tabular}

La implementación de estas estrategias de mejora en las plataformas de compra en línea pueden garantizar un mayor conocimiento del comportamiento y patrón de compra del consumidor. Puesto que las plataformas que conformen todos los elementos que se proporcionan en la Tabla 4, podrán ir adaptándose de acuerdo al análisis del consumidor periódicamente consiguiendo una toma de decisiones efectiva asegurando una mejor aceptación y satisfacción del consumidor. 


\section{DISCUSIÓN}

En el comportamiento del consumidor existe una serie de influencias en el proceso de compra como se describe anteriormente: la motivación y las experiencias. Para esto, una de las influencias más importante está relacionada con la necesidad de existencia de productos, ya que, por ejemplo, cuando no existen los productos en tiendas locales, aumenta la tendencia de consumo en línea. Como ejemplo de ello, el participante 2 opinó "Porque no hay localmente." (P2), el participante 10 comentó "No hay existencia en las tiendas física." (P10) y el participante 5 "a veces en las tiendas no hay lo que buscas y aparte que sale mucho más caro..." (P5); Esta información demuestra que la tendencia de compra en línea podría aumentar al existir escasez de un producto y el alto costo en las tiendas locales. Puesto que en un estudio de la Asociación de Internet MX (2018), demuestra que las compras en línea han aumentado un $17 \%$ más que en el año 2017 , manteniendo un $54 \%$ de usuarios que compran en línea. Sin embargo, este estudio afirma que hay una presencia de las páginas de comercio electrónico en la vida del usuario con un 39\%, al contrario, nuestro estudio demuestra que el $75 \%$ de participantes están de acuerdo que el uso de plataformas de compra en línea cambiaron sus hábitos de compra ya que estas exhiben un mayor catálogo de productos y mejores ofertas.

En definitiva, los consumidores toman en primer lugar esta necesidad como su motivación principal para comprar en sitios de compra en línea, y esto es debido a la influencia de blogs y redes sociales actuales, los cuales han influido de manera notable en el proceso de decisión de compra. En ese sentido, algunos estudios se demuestra la importancia que han tomado las redes sociales por medio de la comunicación y la interacción de información de productos, lo cual afecta el comportamiento de compra favoreciendo la compra en línea (Guardia y Gómez, 2009; Vargas, 2015). Esto es debido a que la publicidad proporcionada en las redes sociales tiene una influencia directa en los aspectos intelectuales, sensitivos y emotivos del consumidor como se ha demostrado en estudios previos (Hsu, Chuan-Chuan y Chiang, 2013). Como ejemplo de ello, el participante 10 opinó: "Las de ropa y todo eso, si fue por publicidad en Facebook, YouTube e Instagram, como yo me la llevo buscando y viendo en esas redes sociales noto esa publicidad..." (P10). Por lo tanto, la influencia de las redes sociales y los blogs pueden influir en las distintas fases del proceso de decisión de compra. Más aún, en este estudio se encontró que los comentarios de los usuarios de los medios sociales tienen un mayor poder de influencia en la decisión de compra que los mensajes de comunicación comercial de cualquier otro medio, en el segmento de población estudiado nacidos entre 1975 y 1995, tomando en cuenta la generación X a la generación Z.

Agregando a lo anterior, los productos y servicios de entretenimiento y de moda son los más consumidos en sitios de compra en línea, puesto que el tiempo entre estímulo de compra (motivación) y la compra (decisión de compra) se ha disminuido en gran medida por causa de la influencia de las redes sociales mencionadas anteriormente, por lo cual los consumidores adquieren más productos en menos tiempo. Sin embargo, un estudio de la Asociación Mexicana de Venta Online (AMVO) demuestra las ventas generadas por el comercio electrónico, donde el sólo el $2 \%$ se genera en México. A pesar de ello, nuestro estudio señala que la población del estado de Sonora, cada vez adquieren mayor confianza para realizar compras en línea de acuerdo a su experiencia en compras previas, las cuales indicaron la existencia de mejores ofertas que en tiendas físicas, variedad de productos y marcas exclusivamente en línea; y de motivación, puesto a las influencias de redes sociales y blogs que promueven una reputación positiva a productos ofrecidos en línea y que las tiendas físicas empiezan a contar con su propio portal de compra en línea. Por otra parte, tanto los sitios web y aplicaciones de compras en línea, han mejorado su proceso de compra, para ofrecer una mayor facilidad y rapidez en la compra de los productos y servicios, lo cual estimulan con mayor intensidad la decisión de compra.

El tipo de perfiles de los participantes seleccionados para el análisis demostraron que las preferencias y patrón de compras son distintos, porque se manifiesta otro tipo de motivaciones y experiencias. El perfil del consumidor en línea generalmente conoce sus posibilidades en el nuevo medio de realizar compras, estas posibilidades de compra se expanden, desde el lugar de conexión, las valoraciones de productos y las recomendaciones personalizadas para la toma de decisiones de compra. Asimismo, las dos clasificaciones de los perfiles de los consumidores son 1) los que desean un ahorro de tiempo y 2) los que desean un ahorro de dinero. Sin embargo, nuestros resultados demuestran el origen del por qué un consumidor se clasifica en uno de los dos grupos y también, el cómo poder anticipar el perfil del consumidor para poder ofrecer un servicio personalizado de acuerdo a las características del usuario en la plataforma de compra en línea. Por otro lado, además de las características abordadas en este trabajo, otros estudios mencionan que una de las características que consideran más importantes para la compra en línea son la confianza y la fiabilidad que percibe el consumidor al momento de realizar la compra en el sitio en línea (Al-Debei, Akroush y Ashouri, 2015; Sánchez-Alzate y Montoya, 2017). Si bien, estas características no se abordan explícitamente como una categoría en este estudio, se consideraron implícitamente dentro del grupo de elementos fundamentales para la generación de estrategias que conlleve a la satisfacción del consumidor en las compras en línea. Por ejemplo, para los participantes que trabajan y adquieren productos y servicios para sus actividades laborales, el factor de mayor importancia es el precio del producto y servicio, por el contrario, si es de tipo personal el factor más importante es el medio de envío y en segundo lugar el precio. De esta manera, los datos obtenidos sobre el comportamiento del consumidor en compras en línea, 
demuestran que los consumidores poseen cada vez más control y es necesario dar a conocer los factores encontrados y sus relaciones que influyen en el patrón y decisión de compra en línea. Asimismo, fue posible generar una teoría con información respecto a aquellos factores de mayor importancia en la decisión y patrón de compra por Internet en los consumidores del estado de Sonora en la compra de productos y servicios en los sectores de tecnología, vestimenta y entretenimiento.

\section{CONCLUSIONES}

Los hallazgos de este estudio demuestran que el enfoque metodológico de la Teoría Fundamentada aplicado en el ámbito de compras por Internet garantizó la identificación de factores clave como la motivación, la experiencia y el comportamiento, los cuales se obtuvieron a partir de una reflexión crítica que permitió relacionar temas de interés para el estudio de comportamiento del consumidor a partir de la realidad observada. Con base en los resultados del estudio, se concluye que el análisis del comportamiento del consumidor puede ayudar a la creación de estrategias para la toma de decisiones efectivas mediante su aplicación en el diseño de plataformas de compra en línea. Estas plataformas podrían proveer al empresario una herramienta tecnológica donde podrá obtener un análisis de sobre el comportamiento de los consumidores para mejorar estrategias de mercadeo y venta.

\section{REFERENCIAS}

Al-Debei, M. M., M. N. Akroush y M. I. Ashouri, Consumer Attitudes towards Online Shopping: The Effects of Trust, Perceived Benefits, and Perceived Web Quality, doi: 10.1108/IntR-05-2014-0146, J. of Internet Research, 25(5), 707-733 (2015)

Asociación de Internet.mx, 14 Estudio sobre los Hábitos de los Usuarios de Internet en México 2018, México (2018)

Asociación Mexicana de Venta Online, Retos y Tendencias del Sector Logístico: "E Commerce y la Última Milla" México 2018, Daqua Strategic Intelligence, México (2018)

Barrera, G. A., Relación de Capacitación con Adopción de Internet y E-Commerce: Diferencias entre Microemprendedores de Chile, doi: http://dx.doi.org/10.4067/S0718-07642017000600008, Revista de Información Tecnológica, 28(6), 61-70 (2017)

Cheung, C.M., I. L. Liu y M. K. Lee, How Online Social Interactions Influence Customer Information Contribution Behavior in Online Social Shopping Communities: a Social Learning Theory Perspective, doi: 10.1002/asi.23340, J. of the Association for Information Science and Technology, 66(12), 2511-2521 (2015)

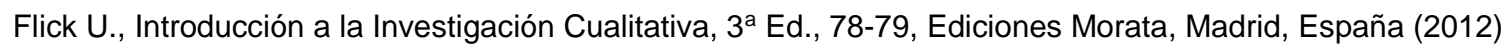

Gallaugher, P.A. J.M., Factors Affecting the Adoption of an Internet-Based Sales Presence for Small Businesses, doi: 10.1080/019722497129287, J. of The Information Society, 13(1), 55-74 (1997)

Gefen, D. y D. W. Straub, The Relative Importance of Perceived Ease of Use in IS Adoption: A Study of E-Commerce Adoption, ISSN: 1536-9323, J. of the Association for Information Systems, 1(8), 1-30 (2000)

Glaser, B. y A. Strauss, The Discovery of Grounded Theory: Strategies for Qualitative Research, Aldine Publishing Company, Chicago, Estados Unidos (1967)

Goldsmith, R.E. y D. Horowitz, Measuring Motivations for Online Opinion Seeking, doi: 10.1080/15252019.2006.10722114, J. of Interactive Advertising, 6(2), 2-14 (2006)

Grandon, E. E. y J. M. Pearson, Electronic Commerce Adoption: an Empirical Study of Small and Medium US Businesses, doi: 10.1016/j.im.2003.12.010, J. of Information \& Management, 42(1), 197-216 (2004)

Guardia, M.L.G. y P.N. Gómez, Bloggers y su Influencia en la Imagen de una Marca, doi: https://doi.org/10.7195/ri14.v7i1.343, Revista ICONO14, Revista Científica de Comunicación y Tecnologías Emergentes, 7(1), 242-252 (2009)

Hausman, A., A Multi-method Investigation of Consumer Motivations in Impulse Buying Behavior, doi: 10.1108/07363760010341045, J. of Consumer Marketing, 17(5), 403-426 (2000)

Homburg, C. y A. Giering, Personal Characteristics as Moderators of the Relationship between Customer Satisfaction and Loyalty-an Empirical Analysis, doi: 10.1002/1520-6793(200101)18:1<43::AID-MAR3>3.0.CO;2-I, J. of Psychology \& Marketing, 18(1), 43-66 (2001)

Hsu, C. L., L. J. Chuan-Chuan y H. S. Chiang, The Effects of Blogger Recommendations on Customers' Online Shopping Intentions, doi: 10.1108/10662241311295782, J. of Internet Research, 23(1), 69-88 (2013)

Joines, J. L., C. W. Scherer y D. A. Scheufele, Exploring Motivations for Consumer Web use and their Implications for ecommerce, ISSN: 0736-3761, J. of Consumer Marketing, 20(2), 90-108 (2003)

Karbasivar, A. y H. Yarahmadi, Evaluating Effective factors on Consumer Impulse Buying Behavior, ISSN: 2222-1387, Asian Journal of Business Management Studies, 2(4), 174-181 (2011)

Locke, K., Grounded theory in Management Research, SAGE Publications Inc., California, Estados Unidos (2001)

Olson, J. S. y G. M. Olson, i2i trust in e-commerce, doi: 10.1145/355112.355121, Communications of the ACM, 43(12), 41-44 (2000) 
Pavlou, P. A. y M. Fygenson, Understanding and Predicting Electronic Commerce Adoption: An Extension of the Theory of Planned Behavior, doi: 10.2307/25148720, J. of Management Information Systems Quarterly, 30(1), 115-143 (2006)

Sánchez-Alzate, J. A. y R. L. A. Montoya, La Confianza como Elemento Fundamental en las Compras a través de Canales de Comercio Electrónico: Caso de los Consumidores en Antioquia, doi: 10.15446/innovar.v27n64.62365, Revista Innovar, 27(64), 11-22 (2017)

Solomon, M. R., D. W. Dahl y otros tres autores, Consumer behavior: Buying, Having, and Being, 6ª Ed., Pearson, Toronto, Canada (2014)

Spiggle, S., Analysis and Interpretation of Qualitative Data in Consumer Research, doi: 10.1086/209413, J. of Consumer Research, 21(3), 491-503 (1994)

Strauss, A. y J. Corbin, Basics of Qualitative research: Techniques and Procedures for Developing Grounded Theory, Thousand Oaks, California, Saga Publication (1998)

Strauss, A.L., J. Corbin y E. Zimmerman, Bases de la Investigación Cualitativa: Técnicas y Procedimientos para Desarrollar la Teoría Fundamentada, $1^{a}$ Ed., Editorial Universidad de Antioquia, Medellín, Colombia (2002)

Valles, M.S., Entrevistas Cualitativas, $2^{\mathrm{a}}$ Ed., 68, Centro de Investigaciones Sociológicas, Madrid, España (2009)

Vargas, S.A.M., Analizar la Influencia de los Mensajes en las Redes Sociales de Marcas de Prendas de Vestir, sobre la Decisión de Compra en Mujeres de 18 a 30 años de la Ciudad de Cali, Tesis de Licenciatura, Universidad Autónoma de Occidente, Facultad de Comunicación Social y Periodismo (2015)

Westbrook, R. A. y R. L. Oliver, The Dimensionality of Consumption Emotion Patterns and Consumer Satisfaction, doi: 10.1086/209243, J. of Consumer Research, 18(1), 84-91 (1991)

Zarco, A.I.J. y M.P.M. Ruiz, La Influencia de las TIC en la Distribución Comercial: Implicaciones Estratégicas para la Gestión Promocional Minorista, ISSN-e: 1681-5645, CTS+ I: Rev. Iberoamericana de Ciencia, Tecnología, Sociedad e Innovación, (7), 9 (2006)

Zhou, L., L. Dai y D. Zhang, Online Shopping Acceptance Model-A Critical Survey of Consumer Factors in Online Shopping, ISSN: 1526-6133, J. of Electronic Commerce Research, 8(1), 41-62 (2007) 